\title{
CURVA DE APRENDIZADO NA FUNDOPLICATURA LAPAROSCÓPICA DURANTE A RESIDÊNCIA MÉDICA EM CIRURGIA GERAL
}

\section{LEARNING CURVE IN THE LAPAROSCOPIC FUNDOPLICATION DURING THE GENERAL SURGERY RESIDENCY PROGRAM}

\author{
William Kondo, AsCBC- PR ${ }^{1}$; Mariana Jorge Garcia²; Flávio Hueta Ivano \\ Luis Carlos von Bahten, TCBC- PR ${ }^{4}$; Rogério Takeshi Miyake, ACBC-PR ${ }^{5}$; Benjamin Smaniotto, TCBC-PR
}

\begin{abstract}
RESUMO: Objetivo: A fundoplicatura laparoscópica a $360^{\circ}$ é o tratamento de escolha para pacientes com doença do refluxo gastroesofágico que não respondem bem ao tratamento clínico. Este artigo demonstra a curva de aprendizado durante a Residência Médica em Cirurgia Geral. Método: Foi realizada uma análise retrospectiva de 60 pacientes submetidos à fundoplicatura laparoscópica durante o período de março a outubro de 2005. Os pacientes foram divididos em dois grupos: 30 casos iniciais que foram comparados aos 30 casos subseqüentes. As variáveis analisadas incluíram: tempo operatório, taxa de conversão, complicações e tempo de hospitalização. Resultados: A fundoplicatura laparoscópica foi realizada em 18 homens e 42 mulheres, com idade média de 48,3 anos. Comparando os dois grupos, houve diferença estatisticamente significativa no tempo operatório total ( 92,7 vs. 76,7 minutos, $p=0,003)$, no tempo operatório para a operação de Nissen-Rossetti $(86,7$ vs. 68,4 minutos, $p=0,00006)$ e no tempo para a liberação dos vasos gástricos curtos (22 vs. 13,1 minutos, $p=0,00005$ ). As complicações intra-operatórias foram maiores no primeiro grupo de pacientes, mas a diferença não foi estatisticamente significativa $(p=0,2)$. Todos os procedimentos foram concluídos com sucesso por vídeo-laparoscopia em ambos os grupos, e a mortalidade foi nula nesta série. A alta hospitalar ocorreu em média no primeiro dia de pós-operatório em ambos os grupos $(p=0,06)$. Conclusão: A fundoplicatura laparoscópica pode ser realizada com segurança por um cirurgião em formação, sob supervisão direta de um titular, com mínima morbidade para os pacientes. A curva de aprendizado foi demonstrada, avaliando-se principalmente, o tempo operatório do procedimento (Rev. Col. Bras. Cir. 2006; 33(2): 96-100).
\end{abstract}

Descritores: Refluxo gastroesofágico; Procedimentos cirúrgicos do sistema digestório/ métodos; Fundoplicatura; Laparoscopia; Aprendizado; Internato e residência; Especialidades cirúrgicas/ educação.

\section{INTRODUÇÃO}

A doença do refluxo gastroesofágico é muito comum e a maioria dos pacientes são tratados clinicamente com inibidores de bomba de prótons e agentes pró-cinéticos ${ }^{1}$. Cerca de $20 \%$ dos pacientes que desenvolvem complicações ou apresentam recorrência dos sintomas após a interrupção do uso da medicação necessitam de tratamento cirúrgico ${ }^{2,3}$.

O tratamento cirúrgico por via aberta ou por laparoscopia da doença do refluxo gastro-esofágico por meio da fundoplicatura é efetivo, duradouro e, em alguns aspectos, superior ao tratamento medicamentoso ${ }^{4-8}$. Estudos demonstrando custo reduzido ${ }^{9}$, menor tempo de permanência hospitalar e retorno precoce ao trabalho ${ }^{9-10}$ comparando as fundoplicaturas laparoscópicas com as abertas têm sido relatados, e são em parte responsáveis pela popularização deste procedimento.

A literatura mostra que uma curva de aprendizado de 20 cirurgias por cirurgião e de 50 procedimentos por instituição é necessária para o sucesso da fundoplicatura laparoscópica ${ }^{1-14}$, embora se saiba que a progressão individual do cirurgião ocorre em diferentes velocidades.

\section{MÉTODO}

Foi realizada uma análise retrospectiva dos prontuários de 60 pacientes submetidos à correção cirúrgica laparoscópica da doença do refluxo gastroesofágico durante o período de março a outubro de 2005. Todos os pacientes foram operados pelo mesmo residente de Cirurgia Geral em treinamento, sob supervisão direta do cirurgião titular.

As indicações para a cirurgia anti-refluxo foram: presença de sintomas refratários ao tratamento clínico, identificação de complicações decorrentes da doença do refluxo gastro-esofágico e o desejo do paciente de descontinuar o tratamento clínico ${ }^{1}$. A válvula anti-refluxo à $360^{\circ}$ foi realizada em todos os pacientes, com ou sem liberação dos vasos curtos, técnicas de Nissen e Nissen-Rossetti respectivamente, de acordo com a preferência individual de cada médico titular.

\footnotetext{
1. Residente de Cirurgia do Trauma do Hospital Cajuru.

2. Residente de Cirurgia Geral da Irmandade Santa Casa de Misericórdia de Curitiba.

3. Cirurgião Geral da Irmandade Santa Casa de Misericórdia de Curitiba.

4. Cirurgião Geral do Hospital Universitário Cajuru.

5. Cirurgião Geral da Irmandade Santa Casa de Misericórdia de Curitiba.

6. Chefe do Serviço de Cirurgia Geral do Hospital Universitário Cajuru.

Recebido em 20/10/2005

Aceito para publicação em 22/12/2005
}

Conflito de interesses: nenhum

Fonte de financiamento: nenhuma

Trabalho realizado no Serviço de Cirurgia Geral do Hospital Universitário Cajuru, e no Serviço de Cirurgia Geral da Irmandade Santa Casa de Misericórdia de Curitiba - PR. 


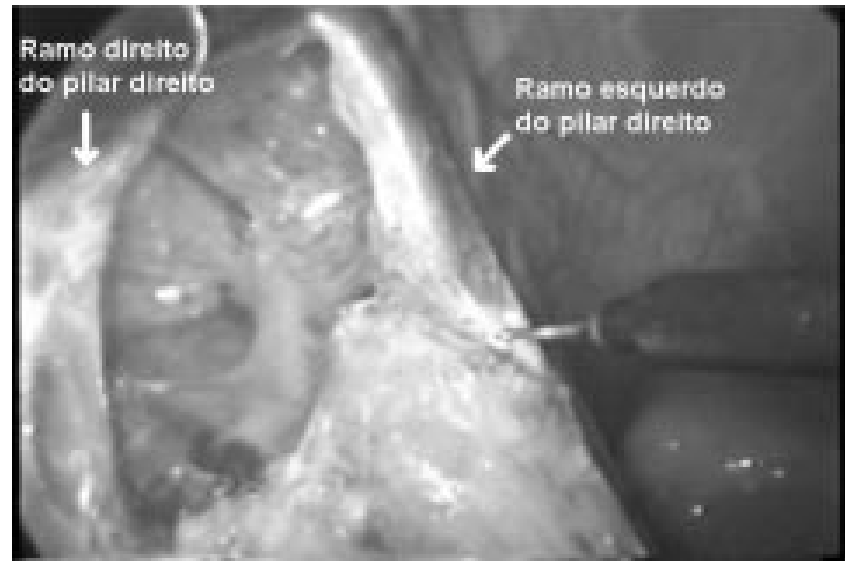

Figura 1 - Dissecção dos pilares diafragmáticos e liberação da membrana freno-esofágica.

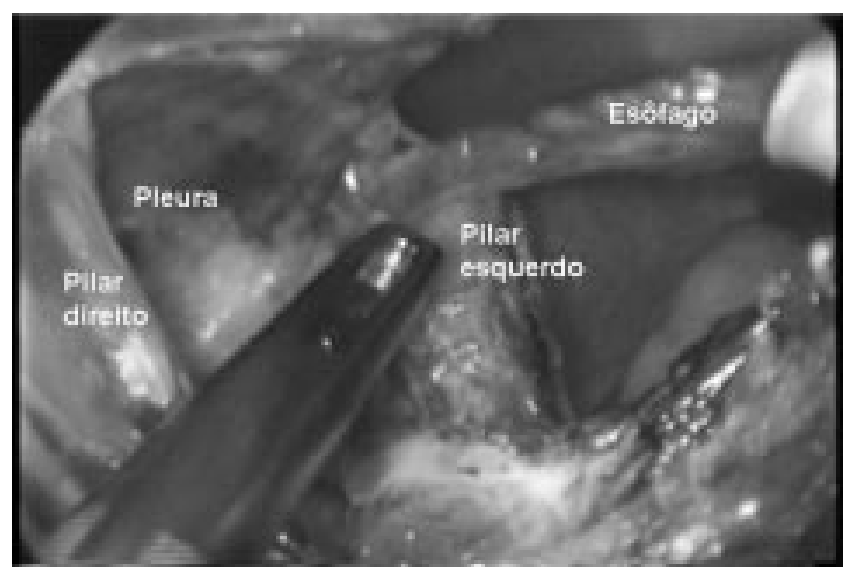

Figura 3 - Dissecção do túnel retroesofágico.

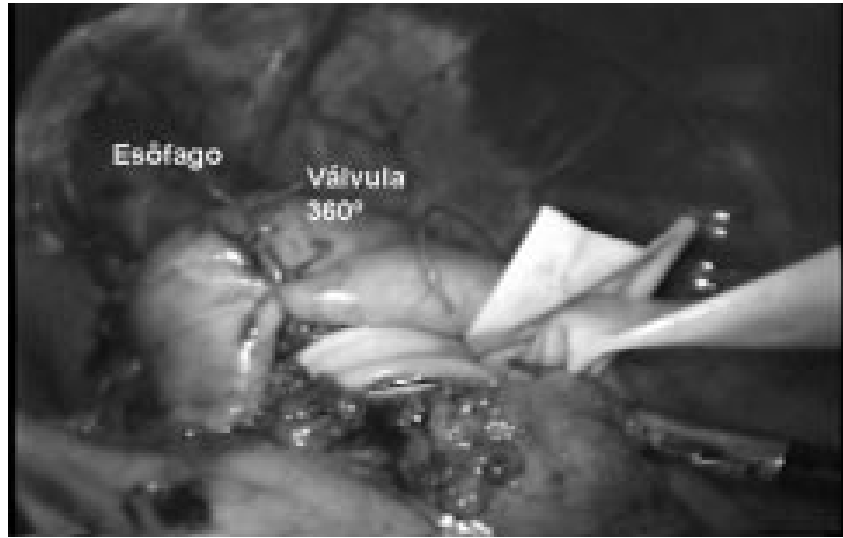

Figura 5 - Aspecto final da válvula.

\section{TÉCNICA OPERATÓRIA}

O paciente estava em decúbito dorsal, sob anestesia geral balanceada. Uma sonda orogástrica era posicionada para esvaziar o estômago e para auxiliar na tração do mesmo. Quando a agulha de Verres estava disponível no material de vídeocirurgia, o pneumoperitônio era confeccionado pela técnica fechada. Naqueles pacientes com operação prévia no andar superior de abdome e na ausência da agulha de Verres, o pneumoperitônio era realizado pela técnica aberta. Os passos da cirurgia estão enumerados a seguir, conforme previamente descritos por Evans et al. ${ }^{15}$.

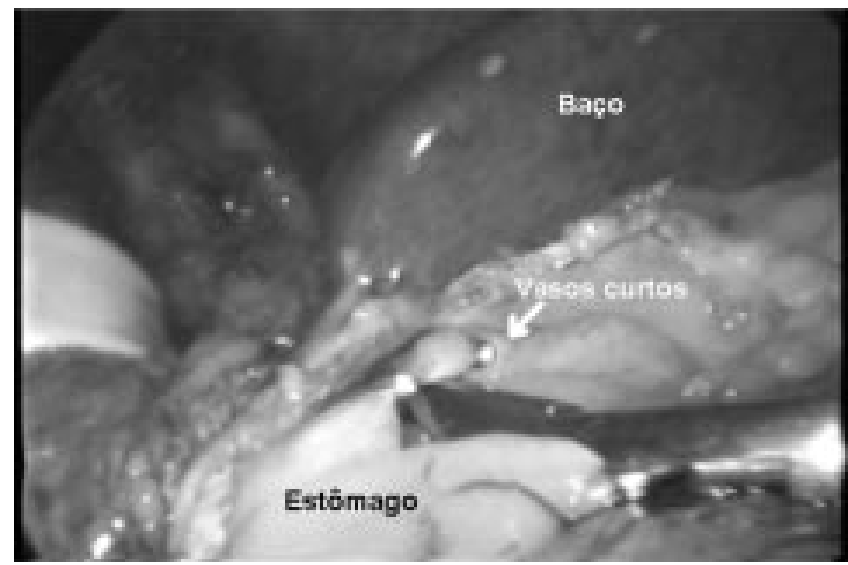

Figura 2 - Liberação dos vasos curtos usando clips metálicos.

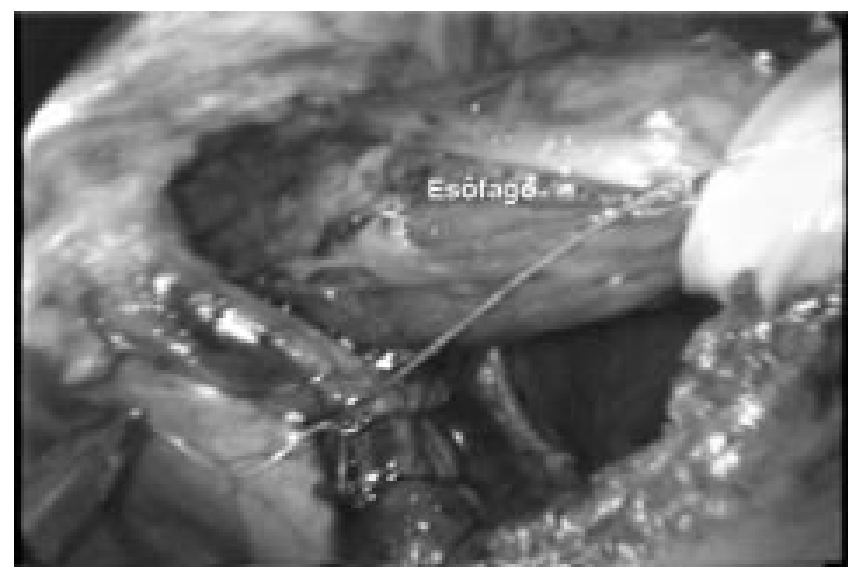

Figura 4 - Aproximação dos pilares diafragmáticos.

1. Posicionamento dos trocartes;

2. Afastamento do fígado;

3. Abertura do ligamento hepato-gástrico;

4. Dissecção dos pilares esofageanos e da membrana freno-esofágica (Figura 1);

5. Alongamento do esôfago abdominal (quando necessário);

6. Mobilização gástrica e ligadura dos vasos gástricos curtos (Figura 2);

7. Dissecção retroesofágica da junção gastroesofágica (Figura 3);

8. Fechamento do hiato esofageano (Figura 4);

9. Confecção da válvula anti-refluxo (Figura 5);

10. Retirada dos trocartes.

O tempo cirúrgico foi mensurado desde a incisão na pele até o completo fechamento da mesma (tempo cirúrgico total). Para fins de análise estatística, o tempo despendido para a liberação dos vasos gástricos curtos nas cirurgias de Nissen foi mensurado separadamente (tempo para liberação dos vasos curtos) do restante do procedimento (tempo para o Nissen-Rossetti), uma vez que apenas a liberação dos vasos gástricos curtos é que diferencia as duas técnicas de fundoplicatura utilizadas em nossos pacientes (Técnica de Nissen $=$ Nissen-Rossetti + liberação dos vasos curtos), e não foi realizada em todos os pacientes. 
Os pacientes foram divididos em dois grupos, sendo que os 30 casos iniciais foram comparados aos 30 casos subseqüentes com o intuito de se estabelecer a curva de aprendizado neste procedimento cirúrgico. As variáveis analisadas incluíram tempo cirúrgico, taxa de conversão, complicações e tempo de hospitalização. O teste $\mathrm{t}$ de Student e o teste exato de Fisher foram utilizados para as comparações estatísticas. $\mathrm{O}$ valor de $p<0,05$ foi considerado estatisticamente significativo. Os dados estão representados pela média \pm desvio padrão.

\section{RESULTADOS}

Durante o período, a fundoplicatura laparoscópica foi realizada em 60 pacientes, incluindo 18 homens e 42 mulheres com idade média de 48,3 $\pm 10,6$ anos (variando de 19 a 71 anos). O índice de massa corporal (IMC) médio, calculado dividindo-se o peso do paciente em quilos pelo quadrado da altura em metros, foi de $27,5 \mathrm{~kg} / \mathrm{m}^{2}$ (variando de 20 a $42 \mathrm{~kg} / \mathrm{m}^{2}$ ).

As Tabelas 1 e 2 comparam os dois grupos de pacientes, demonstrando a curva de aprendizado em nossa série.

O IMC médio foi semelhante nos dois grupos de pacientes.

Três pacientes do segundo grupo apresentavam cirurgia abdominal prévia no andar superior de abdome, sendo um caso de laparotomia exploradora por ferimento transfixante por projétil de arma de fogo, um caso de laparotomia exploradora por úlcera gástrica perfurada e um caso de colecistectomia aberta por incisão mediana. $\mathrm{O}$ tempo cirúrgico desses pacientes foi bastante superior (130, 120 e 130 minutos, respectivamente), contribuindo para elevar o tempo cirúrgico médio dos pacientes do grupo 2. Nos três casos foi possível a liberação das aderências sem lesão das estruturas intra-abdominais e a confecção da cirurgia anti-refluxo sem necessidade de conversão.

Os vasos curtos foram liberados em 28 casos, sendo a maioria deles no grupo $2(67,8 \%)$. Essa diferença não foi levada em consideração uma vez que a opção pela técnica cirúrgica foi uma decisão do cirurgião titular.

O tempo cirúrgico é uma variável útil para se avaliar a experiência do cirurgião ${ }^{1}$, e a diferença entre os dois grupos de pacientes em nossa série foi notável. Houve diferença estatisticamente significativa no tempo cirúrgico total $(92,7$ vs $76,7$ minutos, $p=0,003)$, no tempo cirúrgico para a cirurgia de Nissen-Rossetti (86,7 vs. 68,4 minutos, $p=0,00006$ ) e no tempo para a liberação dos vasos gástricos curtos e mobilização do fundo gástrico ( 22 vs. 13,1 minutos, $p=0,00005$ ).

As complicações intra-operatórias foram maiores no primeiro grupo de pacientes, mas a diferença não foi estatisticamente significativa. Das cinco complicações no primeiro grupo, quatro foram decorrentes de pequeno sangramento durante a liberação dos vasos gástricos curtos e um de lesão de serosa gástrica durante a punção com agulha de Verres. As

Tabela 1 - Comparação entre os 2 grupos de pacientes - utilização do teste t de Student.

\begin{tabular}{lccl}
\hline & Casos 1 a 30 & Casos 31 a 60 & \multicolumn{1}{c}{$\boldsymbol{p}$} \\
\hline IMC em kg/m² (variação) & $27,2 \pm 4,2(20-39)$ & $27,7 \pm 4,3(22-42)$ & 0,67 \\
Tempo cirúrgico total em minutos (variação) $\dagger$ & $92,7 \pm 14,9(75-140)$ & $76,7 \pm 23,1(45-130)$ & 0,003 \\
$\quad$ Nissen-Rossetti $\dagger$ & & & 0,00006 \\
$\quad$ Liberação dos vasos curtos $\dagger$ & $86,7 \pm 12,9(72-140)$ & $68,4 \pm 18,3(45-115)$ & 0,00005 \\
& $22,0 \pm 3,9(15-25)$ & $13,1 \pm 4,8(5-20)$ & \\
Alta hospitalar em dias (variação) & $1,5 \pm 1,0(1$ a 6) & $1,1 \pm 0,3($ a 2$)$ & 0,06 \\
\hline
\end{tabular}

$\dagger$ Houve diferença estatisticamente significativa $(p<0,05)$.

Tabela 2 - Comparação entre os 2 grupos de pacientes - utilização do teste exato de Fisher.

\begin{tabular}{lccc}
\hline & Casos 1 a 30 & Casos 31 a 60 & $\boldsymbol{P}$ \\
\hline Colecistectomia concomitante* & 6 & 1 & 0,4 \\
Cirurgia prévia no andar superior de abdome & 0 & 3 & 0,7 \\
Confecção do pneumoperitônio $\dagger$ & & & $<, 0001$ \\
$\quad$ Técnica aberta & 26 & 5 & \\
$\quad$ Técnica fechada com agulha de Verres & 4 & 25 & 0,009 \\
Liberação dos vasos curtos $\dagger$ & 9 & 2 & 0,2 \\
Complicações intra-operatórias & 5 & 0 & - \\
Conversões & 0 & 0 & - \\
Mortalidade & 0 & 0 & 0,3 \\
Complicações pós-operatórias durante o internamento & 1 & 2 & \\
\hline
\end{tabular}

* O tempo cirúrgico para a realização da colecistectomia não foi incluído no tempo cirúrgico total do procedimento na Tabela 1.

$\dagger$ Houve diferença estatisticamente significativa $(p<0,05)$. 
duas complicações no grupo 2 foram decorrentes de sangramento durante a liberação dos vasos curtos do fundo gástrico.

Todos os procedimentos foram concluídos com sucesso por vídeo-laparoscopia e a mortalidade foi nula nesta série.

Avaliamos apenas as complicações pós-operatórias imediatas, durante o internamento dos pacientes, e observamos um caso de disfagia no grupo 1, que apresentou melhora espontânea, mas necessitou de seis dias de permanência hospitalar.

A alta hospitalar ocorreu em média no primeiro dia de pós-operatório em ambos os grupos.

\section{DISCUSSÃO}

Desde a sua introdução por Nissen ${ }^{16}$, em 1956, a fundoplicatura tornou-se o procedimento anti-refluxo mais utilizado para o tratamento cirúrgico da doença do refluxo gastro-esofágico. A primeira fundoplicatura laparoscópica foi descrita em 1991 por Dallemagne et al. ${ }^{17} \mathrm{e}$ vários trabalhos recentes têm demonstrado sua segurança e efetividade deste método $7,18,19$

A realização da fundoplicatura laparoscópica requer habilidade para se realizar uma cirurgia em múltiplos planos utilizando apenas uma visão bidimensional ${ }^{1}$. $\mathrm{O}$ treinamento para o desenvolvimento desta habilidade e a necessidade de familiarização com os instrumentos laparoscópicos, as técnicas de sutura e as inovações tecnológicas, são os atuais desafios para a realização de cirurgias por via laparoscópica de um modo seguro e eficaz ${ }^{1,20}$.

A aprendizagem da cirurgia é baseada na aquisição de uma série de conhecimentos teóricos sobre fisiopatologia, anatomia patológica, biologia, genética, fisiologia, clínica, técnica cirúrgica, entre outros. Além disso, há necessidade de uma atividade clínica diária com um grupo experiente em um meio hospitalar docente para que sejam desenvolvidas as habilidades e a capacitação técnica necessárias para a realização dos procedimentos cirúrgicos ${ }^{20}$.

A complexidade da aprendizagem da cirurgia laparoscópica é decorrente da ausência de sensibilidade tátil e da noção de profundidade (terceira dimensão), que limitam os gestos e os movimentos comumente realizados durante a cirurgia aberta, da importância do primeiro auxiliar para expor o campo de atuação ao cirurgião, da mínima ajuda que se pode oferecer para que o cirurgião realize os movimentos cirúrgicos, da necessidade de se familiarizar com o material de laparoscopia e da capacidade de resolução dos problemas cotidianos que surgem de sua utilização $\mathrm{O}^{20}$.

As variáveis tradicionalmente utilizadas para demonstrar a curva de aprendizado na fundoplicatura laparoscópica são a taxa de conversão, as complicações e o tempo cirúrgico ${ }^{1,12-14}$. Watson et al. ${ }^{12}$ elucidaram vários aspectos da curva de aprendizado avaliando 280 fundoplicaturas laparoscópicas realizadas por 11 cirurgiões em um estudo em uma mesma instituição. Na sua experiência, a curva de aprendizado chega a um plateau após as primeiras 50 cirurgias para a instituição e após as primeiras 20 cirurgias para cada cirurgião. Este fenômeno tem sido observado tanto em centros de ensino quanto em outros centros não acadêmicos ${ }^{14}$. Soot et al. ${ }^{1}$ obsevaram que o maior aprendizado ocorre nos 25 casos iniciais; no entanto, em um ambiente supervisionado, os residentes parecem adquirir proficiência após cerca de 12 procedimentos.

Em nossa série pudemos reproduzir os resultados obtidos nos estudos previamente citados, demonstrando o efeito da curva de aprendizado na cirurgia de fundoplicatura laparoscópica durante a formação na Residência Médica em Cirurgia Geral. Notamos uma diferença estatisticamente significativa referente ao tempo cirúrgico durante esta curva de aprendizagem (Tabela 1).

Um estudo recente de Ahlberg et al. ${ }^{21}$ pôde demonstrar que a competência do cirurgião "professor" é significativamente o fator mais importante na determinação da habilidade técnica final do cirurgião em treinamento, com variações individuais na curva de aprendizado. As reduzidas taxas de conversões e de complicações observadas neste estudo foram atribuídas à habilidade do cirurgião "professor" em supervisionar e interromper o procedimento quando o cirurgião em treinamento estava apresentando dificuldades. Esses achados são concordantes com observações prévias de que a presença de um cirurgião experiente como supervisor na fundoplicatura laparoscópica pode reduzir a taxa de conversões e de complicações relacionadas ao procedimento ${ }^{12}$.

As complicações observadas em nossa casuística foram sangramento de pouca intensidade, facilmente reparado e lesão da serosa visceral durante a punção com agulha. Todas puderam ser controladas durante o trans-operatório. A liberação dos vasos gástricos curtos nos pareceu o passo cirúrgico com maior risco de sangramento e deve ser realizado com cautela.

A maioria das conversões durante a curva de aprendizado ocorre por razões técnicas durante a dissecção do túnel retroesofágico, a divisão dos vasos gástricos curtos e mobilização do fundo gástrico, o fechamento dos pilares diafragmáticos e a confecção dos pontos da válvula gástrica ${ }^{1}$. Não observamos nenhuma conversão em nossos pacientes, mesmo quando eles apresentavam cirurgia prévia em andar superior de abdome.

Concluímos que a fundoplicatura laparoscópica pode ser realizada com segurança por um cirurgião em formação sob supervisão direta de um titular com experiência em vídeo-cirurgia, com mínima morbidade aos pacientes. A curva de aprendizado realmente existe, e pode ser demonstrada principalmente avaliando-se o tempo cirúrgico do procedimento. Continuamos observando ambulatorialmente esses pacientes e futuramente poderemos comparar os resultados tardios referentes a essa curva de aprendizado. 


\begin{abstract}
Background: The laparoscopic treatment for gastroesophageal reflux disease by total fundoplication is the current surgical treatment of choice after failure of appropriate medical treatment. This article shows the learning curve in the Surgical Residency Program. Methods: We analysed 60 charts of patients submitted to laparoscopic fundoplication between March and October 2005. Patients were separated in 2 groups, and the first 30 cases were compared to the following 30 cases. We collected the following data: operative time, conversion rate, complications ant length of hospitalization. Results: Laparoscopic fundoplication was performed in 18 men and 42 women, with mean age of 48.3 years. Comparing both groups, there was a significant difference in the total operative time (92.7 vs 76.7 minutes, $\mathrm{p}=0,003)$, in the Nissen-Rossetti procedure duration (86.7 vs. 68.4 minutes, $\mathrm{p}=0,00006)$ and in the ligation of short gastric vessels time (22 vs. 13.1 minutes, $\mathrm{p}=0,00005)$. The number of intraoperative complications was bigger in the first group, but the difference was not statistically significant $(\mathrm{p}=0,2)$. No conversion was observed in our series. There was no death. Patients were discharged from the hospital in average at the first postoperative day in both groups $(\mathrm{p}=0,06)$. Conclusion: Laparoscopic fundoplication can be safely performed by residents in their Residency Program with low morbidity rate. We could demonstrate that the effect of the learning curve was more representative related to the operation time.
\end{abstract}

Key words: Gastroesophageal reflux; Digestive system surgical procedures/methods; Fundoplication; Laparoscopy; Learning; Internship and residency; Specialties, surgical/ education.

\section{REFERÊNCIAS}

1. Soot SJ, Eshraghi N, Farahmand M, Sheppard BC, Deveney CW. Transition from open to laparoscopic fundoplication: the learning curve. Arch Surg. 1999; 134(3):278-8; discussion 282.

2. Anvari M, Allen C, Borm A. Laparoscopic Nissen fundoplication is a satisfactory alternative to long-term omeprazole therapy. $\mathrm{Br}$ J Surg. 1995; 82(7):938-42.

3. McKenzie D, Grayson T, Polk HC Jr. The impact of omeprazole and laparoscopy upon hiatal hernia and reflux esophagitis. J Am Coll Surg. 1996;183(4):413-8.

4. DeMeester TR, Bonavina L, Albertucci M. Nissen fundoplication for gastroesophageal reflux disease. Evaluation of primary repair in 100 consecutive patients. Ann Surg. 1986; 204(1):9-20.

5. Spechler SJ. Comparison of medical and surgical therapy for complicated gastroesophageal reflux disease in veterans. The Department of Veterans Affairs Gastroesophageal Reflux Disease Study Group. N Engl J Med. 1992; 326(12):786-92.

6. Anvari M, Allen C. Five-year comprehensive outcomes evaluation in 181 patients after laparoscopic Nissen fundoplication. J Am Coll Surg. 2003;196(1):51-7; discussion 57-8.

7. Booth MI, Jones L, Stratford J, Dehn TC. Results of laparoscopic Nissen fundoplication at 2-8 years after surgery. Br J Surg. 2002; 89(4):476-81.

8. Bammer T, Hinder RA, Klaus A, Klinger PJ. Five- to eight-year outcome of the first laparoscopic Nissen fundoplications. J Gastrointest Surg. 2001; 5(1):42-8.

9. Richards KF, Fisher KS, Flores JH, Christensen BJ. Laparoscopic Nissen fundoplication: cost, morbidity, and outcome compared with open surgery. Surg Laparosc Endosc. 1996; 6(2):140-3.

10. Heikkinen TJ, Haukipuro K, Koivukangas P, Sorasto A, Autio R, Sodervik H, Makela H, Hulkko A. Comparison of costs between laparoscopic and open Nissen fundoplication: a prospective randomized study with a 3-month followup. J Am Coll Surg. 1999; 188(4):368-76.

11. Munro W, Brancatisano R, Adams IP, Falk GL. Complications of laparoscopic fundoplication: the first 100 patients. Surg Laparosc Endosc. 1996; 6(6):421-3.

12. Watson DI, Baigrie RJ, Jamieson GG. A learning curve for laparoscopic fundoplication. Definable, avoidable, or a waste of time? Ann Surg. 1996; 224(2):198-203.

13. Champault GG, Barrat C, Rozon RC, Rizk N, Catheline JM. The effect of the learning curve on the outcome of laparoscopic treatment for gastroesophageal reflux. Surg Laparosc Endosc Percutan Tech. 1999; 9(6):375-81.

14. Voitk A, Joffe J, Alvarez C, Rosenthal G. Factors contributing to laparoscopic failure during the learning curve for laparoscopic Nissen fundoplication in a community hospital. J Laparoendosc Adv Surg Tech A. 1999; 9(3):243-8.

15. Evans SR, Jackson PG, Czerniach DR, Kalan M, Iglesias AR. A stepwise approach to laparoscopic Nissen fundoplication: avoiding technical pitfalls. Arch Surg. 2000; 135(6):723-8.

16. Nissen V. Eine einfache operation zur beeinflussung der refluxoesophagitis. Schweiz Med Wochenschrift. 1956; 86:590-2.

17. Dallemagne B, Weerts JM, Jehaes C, Markiewicz S, Lombard R. Laparoscopic Nissen fundoplication: preliminary report. Surg Laparosc Endosc. 1991; 1(3):138-43.

18. Dallemagne B, Weerts JM, Jeahes C, Markiewicz S, Lombard R. Results of laparoscopic Nissen fundoplication. Hepatogastroenterology. 1998; 45(23):1338-43.

19. Laine S, Rantala A, Gullichsen R, Ovaska J. Laparoscopic vs conventional Nissen fundoplication. A prospective randomized study. Surg Endosc. 1997; 11(5):441-4.

20. Delgado F, Gómez-Abril S, Montalvá E, et al. Formación del residente en cirugía laparoscópica: un recto atual. Cir Esp. 2003; 74(3):134-8

21. Ahlberg G, Kruuna O, Leijonmarck CE, Ovaska J, Rosseland A, Sandbu R, Stromberg C, Arvidsson D. Is the learning curve for laparoscopic fundoplication determined by the teacher or the pupil? Am J Surg. 2005; 189(2):184-9.

Endereço para correspondência:

William Kondo

Avenida Getúlio Vargas, 3163 ap. 21

80240-041- Curitiba - Paraná

e-mail:williamkondo@yahoo.com

Como citar este artigo:

Kondo W, Garcia MJ, Ivano FH, von Bahten LC, Miyake RT, Smaniotto B. Curva de aprendizado na fundoplicatura laparoscópica durante a residência médica em cirurgia geral. Rev Col Bras Cir. [serial on the Internet] 2006 Mar-Apr; 33(2). Available from URL: http:www.scielo.br/rcbc 